\title{
New classes at Specific Age: Properties and Testing hypotheses
}

\author{
M.A.W. Mahmoud ${ }^{1}$, M.E. Moshref ${ }^{1}$, A.M. Gadallah ${ }^{1}$, and A.I. Shawky ${ }^{2}$ \\ ${ }^{1}$ Department of Mathematics, Faculty of Science, Al-Azhar University, \\ Nasr City (11884), Cairo, Egypt \\ ${ }^{2}$ Department of Statistics, Faculty of Sciences, King Abdulaziz University, \\ Saudi Arabia \\ mawmahmoud11@yahoo.com,mmoshrefy@yahoo.com, \\ alaadean_mag@yahoo.com,aishawky@yahoo.com
}

Received 25 May 2012

Accepted 20 February 2013

\begin{abstract}
In this article we introduce new classes of life distributions namely new better (worse) than used in expectation at specific age $t_{0} N B U E-t_{0}\left(N W U E-t_{0}\right)$ and harmonic new better (worse) than used in expectation at specific age $t_{0} H N B U E-t_{0}\left(H N W U E-t_{0}\right)$. The closure properties under various reliability operations such as convolution, mixture, mixing and the homogeneous Poisson shock model of these classes are studied. Furthermore, nonparametric tests are proposed to test exponentiality versus the $N B U E-t_{0}$ and $H N B U E-t_{0}$ classes. The critical values and the powers of this tests are calculated to assess the performance of the tests. It is shown that the proposed tests have high efficiencies for some commonly used distributions in reliability. Sets of real data are used as examples to elucidate the use of the proposed tests for practical problems.
\end{abstract}

Keywords: Convolution, Mixture, U-statistic, Hypothesis test, Homogeneous Poisson shock model.

AMS Subject Classification: 60K10,62E10.

\section{Introduction}

Certain classes of life distributions and their variations have been introduced in reliability, the applications of these classes of life distributions can be seen in engineering, social, biological science, maintenance and biometrics. Therefore, statisticians and reliability analysts have shown a growing interest in modeling survival data using classifications of life distributions based on some aspects of aging. For example Barlow and Proschan (1981), Deshpand et al. (1986) and Cao and Wang (1991), gave definitions of several classes of distributions, $N B U, N B U(2)$ and $N B U E$. Rolski (1975) introduced the HNBUE (harmonic new better than used in expectation) class, subsequently studied by Klefsjo (1982). Testing exponentiality versus the classes of life distributions has been a good deal of attention. For testing against IFR class we refer to Barlow and Proschan (1981) and Ahmed (1975), several other followed. For testing IFRA we refer to Ahmed (1994) and Deshpande (1983), while testing versus NBU are discussed by Hollander and proschan (1972), Koul (1977), Kumazawa (1983) and Ahmed (1994). Mahmoud et al. (2003, 2005, 2007) for NRBU, RNBU and HNRBUE 
M.A.W. Mahmoud et al.

classes. Testing versus NBUL are discussed by Diab et al. (2009) and Diab (2010). Hollander and Proschan (1975), Koul and Susarla (1980), Klefsjoe (1983) and Borges et al. (1984) for (NBUE) class. Finally testing versus $H N B U E$ can be found in the work of Klefsjoe (1983), Basu and Ebrahim (1985), Ahmed (1995) and Hendi et al. (1998) among others. Statisticians and reliability analysts studied some aging classes of life distributions at specific age from various points of view. For more details we refer to Hollander et al. (1986), Ebrahimi and Habbibullah (1990), Ahmed (1998) and Pandit and Anuradha (2007) for $N B U-t_{0}$ and Elbatal (2007) for $N B U C-t_{0}$ and $N B U(2)-t_{0}$.

In this article we introduce new classes of life distribution at specific age $t_{0}$.

Definition 1.1. $X$ is new better than used in expectation at specific age $t_{0}$ (denoted by $X \in N B U E-t_{0}$ ) if

$$
\int_{0}^{\infty} \bar{F}\left(x+t_{0}\right) d x \leqslant \mu \bar{F}\left(t_{0}\right) \text { for all } t_{0} \geqslant 0 .
$$

Definition 1.2. $X$ is harmonic new better than used in expectation at specific age $t_{0}$ (denoted by $\left.X \in H N B U E-t_{0}\right)$ if

$$
\int_{0}^{\infty} \bar{F}\left(x+t_{0}\right) d x \leqslant \mu e^{-t_{0} / \mu} \text { for all } t_{0} \geqslant 0 .
$$

One can note that

$$
N B U \Rightarrow N B U-t_{0} \Rightarrow N B U E-t_{0} \Rightarrow H N B U E-t_{0} .
$$

In the current investigation, Preservation under convolution, mixture, mixing and the homogeneous Poisson shock model of the new classes are discussed in Section 2. In Section 3 we present a procedure to test $X$ is exponential versus it is $N B U E-t_{0}$ and not exponential. Another test is constructed to $H N B U E-t_{0}$ in Section 4. In Section 5, the Pitman asymptotic efficiencies for the two proposed tests are calculated. Monte Carlo null distribution critical points and the power estimates are simulated in Section 6. Finally numerical examples is presented in Section 7.

\section{Closure Properties}

In this section some properties of our classes are introduced under convolution, mixture, mixing and the shock model in homogeneous case.

Theorem 2.1. The NBUE- $t_{0}$ class is preserved under convolution.

Proof. Suppose that $F_{1}$ and $F_{2}$ are two independent $N B U E-t_{0}$ lifetime distributions then their convolution is given by:

$$
\bar{F}=\int_{0}^{\infty} \bar{F}_{1}(z-y) d F_{2}(y) .
$$

Therefore:

$$
\begin{aligned}
\int_{0}^{\infty} \bar{F}\left(x+t_{0}\right) d x & =\int_{0}^{\infty} \int_{0}^{\infty} \bar{F}_{1}\left(x+t_{0}-u\right) d F_{2}(u) d x \\
& =\int_{0}^{\infty} \int_{0}^{\infty} \bar{F}_{1}\left(x+t_{0}-u\right) d x d F_{2}(u) .
\end{aligned}
$$


Since $F_{1}$ is $N B U E-t_{0}$ then

$$
\begin{aligned}
\int_{0}^{\infty} \bar{F}\left(x+t_{0}\right) d x & \leqslant \int_{0}^{\infty} \int_{0}^{\infty} \bar{F}_{1}(x-u) \bar{F}_{1}\left(t_{0}\right) d x d F_{2}(u) \\
& =\bar{F}_{1}\left(t_{0}\right) \int_{0}^{\infty} \bar{F}(x) d x,
\end{aligned}
$$

by using $\bar{F}_{i}(z) \leqslant \bar{F}(z)$ for $i=1,2$, we get

$$
\int_{0}^{\infty} \bar{F}\left(x+t_{0}\right) d x \leqslant \bar{F}\left(t_{0}\right) \int_{0}^{\infty} \bar{F}(x) d x .
$$

Which complete the proof.

The following example is presented to show that $N W U E-t_{0}$ class is not preserved under convolution.

Example 2.1. The convolution of the exponential distribution $F(x)=1-e^{-x}$ with itself yields the gamma distribution of order 2: $G(x)=1-(1+x) e^{-x}$, with strictly increasing failure rate. Thus $G(x)$ is not $N W U E-t_{0}$.

Theorem 2.2. The HNBUE- $t_{0}$ class is preserved under convolution.

Proof. Suppose that $F_{1}$ and $F_{2}$ are two independent $H N B U E$ - $t_{0}$ lifetime distributions then their convolution is given by:

$$
\bar{F}=\int_{0}^{\infty} \bar{F}_{1}(z-y) d F_{2}(y) .
$$

Therefore:

$$
\begin{aligned}
\int_{0}^{\infty} \bar{F}\left(x+t_{0}\right) d x & =\int_{0}^{\infty} \int_{0}^{\infty} \bar{F}_{1}\left(x+t_{0}-u\right) d F_{2}(u) d x \\
& =\int_{0}^{\infty} \int_{0}^{\infty} \bar{F}_{1}\left(x+t_{0}-u\right) d x d F_{2}(u) .
\end{aligned}
$$

Since $F_{1}$ is $H N B U E-t_{0}$ then

$$
\begin{aligned}
\int_{0}^{\infty} \bar{F}\left(x+t_{0}\right) d x & \leqslant \int_{0}^{\infty} \int_{0}^{\infty} e^{-t_{0} / \mu} \bar{F}_{1}(x-u) d x d F_{2}(u) \\
& =e^{-t_{0} / \mu} \int_{0}^{\infty} \int_{0}^{\infty} \bar{F}_{1}(x-u) d F_{2}(u) d x \\
& =e^{-t_{0} / \mu} \int_{0}^{\infty} \bar{F}(x) d x .
\end{aligned}
$$

Which complete the proof.

The following theorem is presented to show that $H N W U E-t_{0}$ class is preserved under convolution.

Theorem 2.3. The HNWUE- $t_{0}$ class is preserved under convolution.

Proof. The proof is obtained by reversing the inequality in the last proof. 
M.A.W. Mahmoud et al.

The following example shows that the $N B U E-t_{0}$ class is not preserved under mixtures.

Example 2.2. Let $\bar{F}_{\alpha}(x)=e^{-\alpha x}$ and $\bar{G}(x)=\int_{0}^{\infty} \bar{F}_{\alpha}(x) e^{-\alpha} d \alpha=(x+1)^{-1}$. Then the failure rate function is $r_{g}(x)=(x+1)^{-1}$, which is strictly decreasing thus $\bar{G}(x)$ is not NBUE-t $t_{0}$.

The following theorem is stated and proved to show that the $N W U E-t_{0}$ class is preserved under mixture.

Theorem 2.4. The NWUE- $t_{0}$ class is preserved under mixture.

Proof. Suppose $F(x)$ is the mixture of $F_{\alpha}$, where each $F_{\alpha}$ is $N W U E-t_{0}$, since

$$
\bar{F}(x)=\int_{0}^{\infty} \bar{F}_{\alpha}(x) d G(\alpha),
$$

then

$$
\begin{aligned}
\int_{0}^{\infty} \bar{F}\left(x+t_{0}\right) d x & =\int_{0}^{\infty} \int_{0}^{\infty} \bar{F}_{\alpha}\left(x+t_{0}\right) d G(\alpha) d x \\
& =\int_{0}^{\infty} \int_{0}^{\infty} \bar{F}_{\alpha}\left(x+t_{0}\right) d x d G(\alpha) .
\end{aligned}
$$

Since $F_{\alpha}$ is $N W U E-t_{0}$ then

$$
\int_{0}^{\infty} \int_{0}^{\infty} \bar{F}_{\alpha}\left(x+t_{0}\right) d x d G(\alpha) \geqslant \int_{0}^{\infty} \int_{0}^{\infty} \bar{F}_{\alpha}\left(t_{0}\right) \bar{F}_{\alpha}(x) d x d G(\alpha) .
$$

Upon using Chebyschev inequality for similarity ordered functions we get

$$
\begin{aligned}
& \int_{0}^{\infty} \int_{0}^{\infty} \bar{F}_{\alpha}\left(t_{0}\right) \bar{F}_{\alpha}(x) d G(\alpha) d x \geqslant \\
& \int_{0}^{\infty}\left\{\int_{0}^{\infty} \bar{F}_{\alpha}\left(t_{0}\right) d G(\alpha) \cdot \int_{0}^{\infty} \bar{F}_{\alpha}(x) d G(\alpha)\right\} d x .
\end{aligned}
$$

Upon using (2.1), (2.2) and (2.3) the proof is completed.

The following theorem is stated and proved to show that the $H N B U E-t_{0}$ class is preserved under mixture.

Theorem 2.5. The HNBUE- $t_{0}$ class is preserved under mixture.

Proof. Suppose $F(x)$ is the mixture of $F_{\alpha}$, where each $F_{\alpha}$ is $H N B U E-t_{0}$ then,

$$
\begin{aligned}
\int_{0}^{\infty} \bar{F}\left(x+t_{0}\right) d x & =\int_{0}^{\infty} \int_{0}^{\infty} \bar{F}_{\alpha}\left(x+t_{0}\right) d G(\alpha) d x \\
& =\int_{0}^{\infty} \int_{0}^{\infty} \bar{F}_{\alpha}\left(x+t_{0}\right) d x d G(\alpha) .
\end{aligned}
$$

Since $F_{\alpha}$ is $H N B U E-t_{0}$ then

$$
\begin{aligned}
\int_{0}^{\infty} \int_{0}^{\infty} \bar{F}_{\alpha}\left(x+t_{0}\right) d x d G(\alpha) & \leqslant \int_{0}^{\infty} \int_{0}^{\infty} e^{-t_{0} / \mu} \bar{F}_{\alpha}(x) d x d G(\alpha) \\
& =e^{-t_{0} / \mu} \int_{0}^{\infty} \int_{0}^{\infty} \bar{F}_{\alpha}(x) d G(\alpha) d x \\
& =e^{-t_{0} / \mu} \int_{0}^{\infty} \bar{F}(x) d x
\end{aligned}
$$


The following theorem is presented to show that $H N W U E-t_{0}$ class is preserved under mixture.

Theorem 2.6. The HNWUE- $t_{0}$ class is preserved under mixture.

Proof. The proof is obtained by reversing the inequality in the last proof.

The following example illustrates that the $N B U E-t_{0}$ and $H N B U E-t_{0}$ classes is not preserved under mixing.

Example 2.3. Let $\bar{F}_{1}=e^{-\delta x}$ and $\bar{F}_{2}=e^{-\gamma x}$. Let $\bar{F}=\frac{1}{2} \bar{F}_{1}+\frac{1}{2} \bar{F}_{2}$. It follows that both $\bar{F}_{1}$ and $\bar{F}_{2}$ are $N B U E-t_{0}$ and $H N B U E-t_{0}$ but $\bar{F}$ is neither NBUE- $t_{0}$ nor $H N B U E-t_{0}$.

\subsection{Homogeneous Poisson Shock Model}

Suppose that a device is subjected to sequence shocks occurring randomly in the time according to a Poisson process with constant intensity $\lambda$. Suppose further that the device has probability $\bar{p}_{k}$ of surviving the first $k$ shocks, where $1=\bar{p}_{0} \geqslant \bar{p}_{1} \geqslant \cdots$. Then the survival function of the device is given by

$$
\bar{H}(t)=\sum_{k=0}^{\infty} \bar{p}_{k} \frac{(\lambda t)^{k}}{k !} e^{-\lambda t}
$$

This shock model has been studied by Esary (1969) for IFR, IFRA, DMRL, NBU, and NBUE classes. Klefsjo (1981) for HNBUE and Mahmoud et al. (2009) for NBURFR- $t_{0}$.

Definition 2.1. A discrete distribution $p_{k}, k=0,1, \ldots, \infty$ or its survival probabilities $\bar{p}_{k}, k=$ $0,1, \ldots, \infty$ is said to have discrete new better (worse) than used expectation at specified time $t_{0}$ $\left(N B U E-t_{0}\right)\left(N W U E-t_{0}\right)$ if

$$
\sum_{r=0}^{\infty} \bar{p}_{r+j} \leqslant(\geqslant) \bar{p}_{j} \sum_{r=0}^{\infty} \bar{p}_{r}, \quad j=0,1, \ldots
$$

Definition 2.2. A discrete distribution $p_{k}, k=0,1, \ldots, \infty$ or its survival probabilities $\bar{p}_{k}, k=$ $0,1, \ldots, \infty$ is said to have discrete harmonic new better (worse)than used expectation at specific age $t_{0}\left(H N B U E-t_{0}\right)\left(H N W U E-t_{0}\right)$ if

$$
\sum_{r=0}^{\infty} \bar{p}_{r+j} \leqslant(\geqslant)\left(1-\frac{1}{m}\right)^{j} \sum_{r=0}^{\infty} \bar{p}_{r}, \quad j=0,1, \ldots
$$

Now, let us introduce the following theorems.

Theorem 2.7. If $p_{k}$ is discrete NBUE- $t_{0}$, then $\bar{H}(t)$ given by (2.4) is NBUE- $t_{0}$. 
M.A.W. Mahmoud et al.

Proof. It must be shown that

$$
\int_{0}^{\infty} \bar{H}\left(x+t_{0}\right) d x \leqslant \bar{H}\left(t_{0}\right) \int_{0}^{\infty} \bar{H}(x) d x .
$$

Upon using (2.4), we get

$$
\begin{gathered}
\int_{0}^{\infty} \bar{H}\left(x+t_{0}\right) d x=\int_{0}^{\infty} \sum_{k=0}^{\infty} \bar{p}_{k} \frac{\left[\lambda\left(t_{0}+x\right)\right]^{k}}{k !} e^{-\lambda\left(x+t_{0}\right)} d x \\
=e^{-\lambda t_{0}} \sum_{k=0}^{\infty} \bar{p}_{k} \sum_{r=0}^{k}\left(\begin{array}{l}
k \\
r
\end{array}\right) \frac{\left(\lambda t_{0}\right)^{k-r}}{k !} \int_{0}^{\infty}(\lambda x)^{r} e^{-\lambda x} d x .
\end{gathered}
$$

Integrating by parts yields

$$
\begin{aligned}
& =\frac{e^{-\lambda t_{0}}}{\lambda} \sum_{r=0}^{\infty} \sum_{k=r}^{\infty} \bar{p}_{k} \frac{\left(\lambda t_{0}\right)^{(k-r)}}{(k-r) !} \\
& =\frac{e^{-\lambda t_{0}}}{\lambda} \sum_{r=0}^{\infty} \sum_{j=0}^{\infty} \bar{p}_{j+r} \frac{\left(\lambda t_{0}\right)^{j}}{j !} \\
& \leqslant \frac{e^{-\lambda t_{0}}}{\lambda} \sum_{j=0}^{\infty} \sum_{r=0}^{\infty} \bar{p}_{j} \bar{p}_{r} \frac{\left(\lambda t_{0}\right)^{j}}{j !} \\
& =\bar{H}\left(t_{0}\right) \int_{0}^{\infty} \sum_{r=0}^{\infty} \frac{(\lambda x)^{r}}{r !} \bar{p}_{r} e^{-\lambda x} d x \\
& =\bar{H}\left(t_{0}\right) \int_{0}^{\infty} \bar{H}(x) d x .
\end{aligned}
$$

The proof for the $N W U E-t_{0}$ class is obtained by reversing the inequality.

Theorem 2.8. If $p_{k}$ is discrete HNBUE- $t_{0}$, then $\bar{H}(t)$ given by (2.4) is HNBUE- $t_{0}$.

Proof. Upon using (2.4), we have

$$
\mu=\int_{0}^{\infty} \bar{H}(t) d t=\frac{1}{\lambda} \sum_{k=0}^{\infty} \bar{p}_{k} \int_{0}^{\infty} \frac{(\lambda t)^{k}}{k !} d(\lambda t)=\frac{1}{\lambda} \sum_{k=0}^{\infty} \bar{p}_{k}=\frac{m}{\lambda},
$$

where $\mathrm{m}$ is the mean of the discrete distribution $\bar{p}_{k}$.

It must be shown that

$$
\int_{0}^{\infty} \bar{H}\left(x+t_{0}\right) d x \leqslant e^{-t_{0} / \mu_{H}} \int_{0}^{\infty} \bar{H}(x) d x
$$

Upon using (2.4), we get

$$
\begin{aligned}
\int_{0}^{\infty} \bar{H}\left(x+t_{0}\right) d x & =\int_{0}^{\infty} \sum_{k=0}^{\infty} \bar{p}_{k} \frac{\left[\lambda\left(t_{0}+x\right)\right]^{k}}{k !} e^{-\lambda\left(x+t_{0}\right)} d x \\
& =e^{-\lambda t_{0}} \sum_{k=0}^{\infty} \bar{p}_{k} \sum_{r=0}^{k}\left(\begin{array}{l}
k \\
r
\end{array}\right) \frac{\left(\lambda t_{0}\right)^{k-r}}{k !} \int_{0}^{\infty}(\lambda x)^{r} e^{-\lambda x} d x .
\end{aligned}
$$


Integrating by parts yields

$$
\begin{aligned}
& =\frac{e^{-\lambda t_{0}}}{\lambda} \sum_{r=0}^{\infty} \sum_{k=r}^{\infty} \bar{p}_{k} \frac{\left(\lambda t_{0}\right)^{(k-r)}}{(k-r) !} \\
& =\frac{e^{-\lambda t_{0}}}{\lambda} \sum_{r=0}^{\infty} \sum_{j=0}^{\infty} \bar{p}_{j+r} \frac{\left(\lambda t_{0}\right)^{j}}{j !} \\
& \leqslant \frac{e^{-\lambda t_{0}}}{\lambda} \sum_{j=0}^{\infty} \sum_{r=0}^{\infty}\left(1-\frac{1}{m}\right)^{j} \bar{p}_{r} \frac{\left(\lambda t_{0}\right)^{j}}{j !} \\
& =e^{\frac{-\lambda t_{0}}{m}} \int_{0}^{\infty} \sum_{r=0}^{\infty} \frac{(\lambda x)^{r}}{r !} \bar{p}_{r} e^{-\lambda x} d x \\
& =e^{-t_{0} / \mu_{H}} \int_{0}^{\infty} \bar{H}(x) d x .
\end{aligned}
$$

The proof for the $H N W U E-t_{0}$ class is obtained by reversing the inequality.

\section{Testing in the NBUE- $t_{0}$}

Our goal in this section is to propose a test statistic for testing exponentiality against $N B U E-t_{0}$ class of life distributions. The following is the measure of departure from $H_{0}$

$$
\triangle_{1}=\mu \bar{F}\left(t_{0}\right)-\int_{0}^{\infty} \bar{F}\left(x+t_{0}\right) d x
$$

it is clear that

$$
\int_{0}^{\infty} \bar{F}\left(x+t_{0}\right) d x=\mu-t_{0},
$$

then

$$
\triangle_{1}=\mu \bar{F}\left(t_{0}\right)-\mu+t_{0} .
$$

One can notice that the value of $\triangle_{1}$ under $H_{0}$ equals $\zeta$, where

$$
\zeta=\bar{F}\left(t_{0}\right)+t_{0}-1
$$

Consider $\delta_{1}=\triangle_{1}-\zeta, \delta_{1}$ can be written in the following form

$$
\delta_{1}=\left[\bar{F}\left(t_{0}\right)-1\right] E[X-1] .
$$

Note that under $H_{0}: \delta_{1}=0$, while under $H_{1}: \delta_{1}>0$. 
M.A.W. Mahmoud et al.

To estimate $\delta_{1}$, let $X_{1}, X_{2}, \ldots, X_{n}$ be a random sample from $F$, so the empirical form of $\delta_{1}$ in (3.4) is

$$
\hat{\delta}_{1 n}=\frac{\left[\bar{F}\left(t_{0}\right)-1\right]}{n} \sum_{i=1}^{n}\left[X_{i}-1\right]
$$

To find the limiting distribution of $\hat{\delta}_{1 n}$ we resort to the U-statistic theory. Let

$$
\phi(X)=\left[\bar{F}\left(t_{0}\right)-1\right][X-1],
$$

and define the symmetric kernel

$$
\psi(X)=\sum_{R} \phi\left(X_{i}\right)
$$

where the sum is over all arrangements of $X_{i}$, this leads that $\hat{\delta}_{1 n}$ in (3.5) is equivalent to U-statistic given by

$$
U_{n}=\frac{1}{n} \sum_{R} \psi\left(X_{i}\right)
$$

The next results summarizes the asymptotic normality of $\hat{\delta}_{1 n}$.

\section{Theorem 3.1.}

(i) As $n \rightarrow \infty, \sqrt{n}\left(\hat{\delta}_{1 n}-\delta_{1}\right)$ is asymptotically normal with mean 0 and variance is

$$
\sigma^{2}=\operatorname{Var}\left\{\left[\bar{F}\left(t_{0}\right)-1\right](X-1)\right\} .
$$

(ii) Under $H_{0}$, the variance is reduced to

$$
\sigma_{0}^{2}=\left(e^{-t_{0}}-1\right)^{2}
$$

The proof is emitted because it is clear.

\section{Testing in the $H N B U E-t_{0}$}

The main aim of this section is to propose a test statistic for testing $H_{0}: F$ is exponential versus $H_{1}$ : $F$ belongs to $H N B U E-t_{0}$ class and not exponential. We propose the following measure of departure

$$
\triangle_{2}=\mu e^{-t_{0}}-\int_{0}^{\infty} \bar{F}\left(x+t_{0}\right) d x,
$$

it is clear that

$$
\int_{0}^{\infty} \bar{F}\left(x+t_{0}\right) d x=\mu-t_{0}
$$

then

$$
\triangle_{2}=\mu e^{-t_{0}}-\mu+t_{0}
$$

One can notice that the value of $\triangle_{2}$ under $H_{0}$ equals $\xi$, where

$$
\xi=e^{-t_{0}}+t_{0}-1 \text {. }
$$


Consider $\delta_{2}=\triangle_{2}-\xi, \delta_{2}$ can be written in the following form

$$
\delta_{2}=\left[e^{-t_{0}}-1\right] E[X-1] .
$$

Based on a random sample $X_{1}, X_{2}, X_{3}, \ldots, X_{n}$ from a distribution $F$ an unbiased estimate of $\delta_{2}$ is given by

$$
\hat{\delta}_{2 n}=\frac{\left[e^{-t_{0}}-1\right]}{n} \sum_{i=1}^{n}\left[X_{i}-1\right]
$$

To find the limiting distribution of $\hat{\delta}_{2 n}$. Set

$$
\Phi(X)=\left[e^{-t_{0}}-1\right][X-1] .
$$

Thus, the variance is

$$
\sigma^{2}=\operatorname{Var}[\phi(X)]
$$

Under $H_{0}$, we get

$$
\sigma_{0}^{2}=\left(e^{-t_{0}}-1\right)^{2}
$$

According to $U$-statistic theory (cf. Lee, 1990), Theorem 4.1 is immediate.

\section{Theorem 4.1.}

(i) As $n \rightarrow \infty, \sqrt{n}\left(\hat{\delta}_{2 n}-\delta_{2}\right) \sim N\left(0, \sigma^{2}\right)$, where

$$
\sigma^{2}=\operatorname{Var}\left(\left[e^{-t_{0}}-1\right][X-1]\right)
$$

(ii) Under $H_{0}$ the variance is reduced to $\sigma_{0}^{2}$ in (4.6).

\section{The Pitman Asymptotic Efficiency (PAE)}

To asses how good this procedures are relative to others in the literature we evaluate its Pittman asymptotic efficiency (PAE) for two alternatives in our classes $N B U E-t_{0}$ and $H N B U E-t_{0}$, these are:

1. Linear failure rate family (LFR): $\bar{F}_{\theta}(x)=\exp \left(-x-\frac{\theta}{2} x^{2}\right), x>0, \theta \geqslant 0$.

2. Makeham family: $\bar{F}_{\theta}=\exp \left(-x+\theta\left(x+e^{-x}-1\right)\right), x>0, \theta \geqslant 0$.

The PAE is defined by

$$
\operatorname{PAE}(\delta)=\frac{1}{\sigma_{0}}\left|\frac{d \delta}{d \theta}\right|_{\theta \rightarrow \theta_{0}} .
$$


In the above cases, we can prove that,

$$
\begin{aligned}
& P A E\left(\hat{\delta}_{1}, F\right)=\frac{1}{\sigma_{0}}\left|\bar{F}_{\theta}^{\prime}\left(t_{0}\right)+\bar{F}_{\theta}\left(t_{0}\right) \int_{0}^{\infty} \bar{F}_{\theta}^{\prime}(x) d x-\int_{t_{0}}^{\infty} \bar{F}_{\theta}^{\prime}(x) d x\right|, \\
& P A E\left(\hat{\delta}_{2}, F\right)=\frac{1}{\sigma_{0}}\left|e^{-t_{0}} \int_{0}^{\infty} \bar{F}_{\theta}^{\prime}(x) d x-\int_{t_{0}}^{\infty} \bar{F}_{\theta}^{\prime}(x) d x\right|, \\
& P A E\left(\hat{\delta}_{1}, L F R\right)=\frac{1}{\sigma_{0}}\left|t_{0} e^{-t_{0}}\right|, \\
& \operatorname{PAE}\left(\hat{\delta}_{1}, \text { Makeham }\right)=\frac{1}{\sigma_{0}}\left|\frac{1}{2} e^{-t_{0}}\left(1-e^{-t_{0}}\right)\right|, \\
& \operatorname{PAE}\left(\hat{\delta}_{2}, \text { LFR }\right)=\frac{1}{\sigma_{0}}\left|e^{-t_{0}}\left(\frac{t_{0}^{2}}{2}+t_{0}\right)\right|, \\
& \text { and } \quad \\
& P A E\left(\hat{\delta}_{2}, \text { Makeham }\right)=\frac{1}{\sigma_{0}}\left|\frac{1}{2} e^{-t_{0}}\left(2 t_{0}+e^{-t_{0}}-1\right)\right| .
\end{aligned}
$$

We notice that $t_{0}=0.1$ is the value that maximizes the above values.

We compare the above PAE's at $t_{0}=0.1$ with that of Hollander and Proschan (1975) and Ahmad et al. (1999), and the results are shown in Table 1.

Table 1 shows that our tests outperforms the others tests for the two alternatives.

\section{Monte Carlo Null Distribution Critical Points}

Many practitioners, such as applied statisticians, and reliability analysts are interested in simulated percentiles. Table 2 gives these percentile points of the statistics $\hat{\delta}_{1 n}$ and $\hat{\delta}_{2 n}$ given in (3.5) and (4.5) at $t_{0}=0.1$ and the calculations are based on 1000 simulated samples of sizes $n=2(1) 50$.

\subsection{The Power Estimates}

Tables 3 and 4 show the power estimate of the test statistic $\hat{\delta}_{1 n}$ and $\hat{\delta}_{2 n}$ given in (3.5) and (4.5) respectively at the significant level 0.05 using LFR and Makeham distributions. The estimates are based on 1000 simulated samples for sizes $n=10,20$ and 30 .

From Tables 3 and 4 we can show that our tests have perfect power.

\section{Numerical Examples}

Example 7.1. The following data represent 39 liver cancers patients taken from Elminia cancer center Ministry of Health - Egypt, which entered in (1999). The ordered life times (in years) are:

\begin{tabular}{|l|l|l|l|l|l|l|l|l|l|}
\hline 0.027 & 0.038 & 0.038 & 0.038 & 0.038 & 0.038 & 0.041 & 0.047 & 0.049 & 0.055 \\
\hline 0.055 & 0.055 & 0.055 & 0.055 & 0.063 & 0.063 & 0.066 & 0.071 & 0.082 & 0.082 \\
\hline 0.085 & 0.110 & 0.314 & 0.140 & 0.143 & 0.164 & 0.167 & 0.184 & 0.195 & 0.203 \\
\hline 0.206 & 0.238 & 0.263 & 0.288 & 0.293 & 0.293 & 0.293 & 0.318 & 0.411 & \\
\end{tabular}

It was found that

- $\hat{\delta}_{1 n}=0.509$ and this value greater than the tabulated critical value in Table 1 . Then we accept $H_{1}$ which states that the data has $N B U E-t_{0}$ property. 
- $\hat{\delta}_{2 n}=0.098$ which is greater than the tabulated critical value in Table 1 . Then we accept $H_{1}$ which states that the data set has $H N B U E-t_{0}$ property.

Example 7.2. Consider the well-known Darwin data (Fisher, 1966) that represent the differences in heights between cross- and self-fertilized plants of the same pair grown together in one pot

\begin{tabular}{|c|c|c|c|c|c|c|c|}
\hline 4.9 & -6.7 & 0.8 & 1.6 & 2.3 & 2.8 & 4.1 & 1.4 \\
\hline 2.9 & 0.6 & 5.6 & 2.4 & 7.5 & 6.0 & -4.8 & \multicolumn{1}{|c}{} \\
\cline { 1 - 4 }
\end{tabular}

It was found that

- $\hat{\delta}_{1 n}=0.9$ which is greater than the tabulated critical value in Table 1 . Then we conclude that this data set has $N B U E-t_{0}$ property.

- $\hat{\delta}_{2 n}=-465.079$ and this value less than the tabulated critical value in Table 1 . There is enough evidence to accept $H_{0}$ which states that the data set has exponential property.

\section{Appendix}

Table 1. The PAE's for LFR and Makeham families

\begin{tabular}{|c|c|c|}
\hline Test & LFR & Makeham \\
\hline Hollander-Proschan & 0.8660 & 0.2886 \\
\hline Ahmad et al . & 0.7490 & 0.2800 \\
\hline$\delta_{1}$ & 0.951 & 0.4524 \\
\hline$\delta_{2}$ & 0.998 & 0.4984 \\
\hline
\end{tabular}


M.A.W. Mahmoud et al.

Table 2. Critical values of statistic $\hat{\delta}_{1 n}$ and $\hat{\delta}_{2 n}$ at $t_{0}=0.1$

\begin{tabular}{|c|c|c|c|c|c|c|}
\hline$n$ & 0.01 & 0.05 & 0.10 & 0.90 & 0.95 & 0.99 \\
\hline 2 & -0.214 & -0.137 & -0.088 & 0.071 & 0.080 & 0.090 \\
\hline 3 & 0.178 & -0.103 & -0.075 & 061 & 070 & .084 \\
\hline 4 & -0.144 & -0.087 & -0.065 & 0.053 & 0.062 & 0.076 \\
\hline 5 & -0.126 & -0.079 & -0.060 & 0.048 & 0.057 & 0.069 \\
\hline 6 & -0.115 & -0.074 & -0.051 & .045 & .054 & 0.068 \\
\hline 7 & -0.102 & -0.065 & -0.047 & 043 & 051 & .066 \\
\hline 8 & -0.089 & -0.060 & -0.046 & 0.041 & 0.048 & 0.060 \\
\hline 9 & -0.086 & -0.053 & -0.042 & 0.038 & 0.045 & 0.057 \\
\hline 10 & -0.080 & -0.053 & -0.039 & 037 & .044 & 0.054 \\
\hline 11 & -0.084 & -0.053 & -0.037 & 36 & .043 & .052 \\
\hline 12 & -0.079 & -0.046 & -0.035 & .036 & 0.042 & .054 \\
\hline 13 & -0.075 & -0.044 & -0.033 & 0.034 & .041 & 0.053 \\
\hline 14 & -0.072 & -0.046 & -0. & 0.032 & 0.039 & 0.049 \\
\hline 15 & -0.0 & -0.044 & -0 . & 30 & 36 & 049 \\
\hline 16 & -0.065 & -0.042 & -0. & 31 & 37 & 048 \\
\hline 17 & -0.063 & -0.041 & -0.030 & .029 & 036 & 0.047 \\
\hline 18 & -0.060 & -0.039 & -0.028 & 0.030 & 036 & 0.047 \\
\hline 19 & -0.061 & -0.038 & -0. & 0.028 & 0.034 & 0.044 \\
\hline 20 & -0.0 & -0 . & -0 . & 29 & & 945 \\
\hline 21 & -0.059 & -0 . & -0 & 27 & 32 & .041 \\
\hline 22 & -0.0 & -0. & -0 & 0.027 & 34 & 0.043 \\
\hline 23 & -0.054 & -0.0 & -0. & 0.025 & 0.032 & 0.043 \\
\hline 24 & -0.0 & -0 & -0 & 26 & 32 & 0.041 \\
\hline 25 & -0.0 & -0 . & -0 & 26 & 31 & 041 \\
\hline 26 & -0.050 & -0.0 & $-0 .($ & 24 & 0.029 & 0.039 \\
\hline 27 & -0.050 & -0.0 & -0.025 & 0.024 & 0.030 & 0.040 \\
\hline 28 & -0.0 & -0. & -0 . & 3 & 28 & 0.038 \\
\hline 29 & -0.0 & -0 . & -0 . & 4 & 30 & 39 \\
\hline 30 & -0.0 & -0 . & -0 . & 21 & 27 & 0.037 \\
\hline 32 & -0.042 & -0. & -0 & & & 0.037 \\
\hline 33 & -0.0 & -0.0 & -0. & & 0.027 & 0.035 \\
\hline 34 & -0.0 & -0. & -0. & 1 & 0.027 & 0.036 \\
\hline 35 & -0.0 & -0 . & -0 . & 21 & 26 & 0.033 \\
\hline 36 & -0.041 & -0.0 & -0 & & 0.026 & 0.033 \\
\hline 37 & -0.042 & -0.027 & -0.020 & 0.020 & 0.025 & 0.033 \\
\hline 38 & -0. & -0.0 & -0.020 & 0.020 & 0.026 & 0.035 \\
\hline 39 & -0.0 & -0 . & -0 . & 20 & 0.025 & 0.031 \\
\hline 40 & -0.0 & -0 & -0 . & & & 0.033 \\
\hline 41 & -0.0 & -0.026 & -0.020 & & 0.023 & 0.031 \\
\hline 42 & -0.037 & -0.0 & -0 & & 0.023 & 0.033 \\
\hline 43 & -0.0 & -0.0 & -0. & & 0.023 & 0.032 \\
\hline 44 & -0.0 & -0. & -0 & & 0.023 & 0.032 \\
\hline 45 & -0.037 & -0.026 & -0.019 & 0.019 & 0.024 & 0.030 \\
\hline 46 & -0.0 & -0.026 & -0. & 0.019 & 0.023 & 0.030 \\
\hline 47 & -0.0 & & & & & 0.030 \\
\hline 48 & -0.039 & -0.0 & -0 . & & 0.022 & 0.029 \\
\hline 49 & -0.037 & -0.024 & -0.018 & & 0.023 & 0.029 \\
\hline 50 & -0.035 & -0.023 & -0.018 & 0.017 & 0.022 & 0.029 \\
\hline
\end{tabular}

Published by Atlantis Press

Copyright: the authors 
Table 3. Power estimates using $\alpha=0.05$ at $t_{0}=0.1$

\begin{tabular}{|c|c|c|c|c|c|}
\hline & $n$ & $\theta=1$ & $\theta=2$ & $\theta=3$ & $\theta=4$ \\
\hline \multirow{3}{*}{ LFR } & 10 & 1.000 & 1.000 & 1.000 & 1.000 \\
& 20 & 1.000 & 1.000 & 1.000 & 1.000 \\
& 30 & 1.000 & 1.000 & 1.000 & 1.000 \\
\hline \multirow{3}{*}{ Makeham } & 10 & 1.000 & 1.000 & 1.000 & 1.000 \\
& 20 & 1.000 & 1.000 & 1.000 & 1.000 \\
& 30 & 1.000 & 1.000 & 1.000 & 1.000 \\
\hline
\end{tabular}

Table 4. Power estimates using $\alpha=0.05$ at $t_{0}=0.1$

\begin{tabular}{|c|c|c|c|c|c|}
\hline & $n$ & $\theta=1$ & $\theta=2$ & $\theta=3$ & $\theta=4$ \\
\hline \multirow{3}{*}{ LFR } & 10 & 1.000 & 1.000 & 1.000 & 1.000 \\
& 20 & 1.000 & 1.000 & 1.000 & 1.000 \\
& 30 & 1.000 & 1.000 & 1.000 & 1.000 \\
\hline \multirow{3}{*}{ Makeham } & 10 & 1.000 & 1.000 & 1.000 & 1.000 \\
& 20 & 1.000 & 1.000 & 1.000 & 1.000 \\
& 30 & 1.000 & 1.000 & 1.000 & 1.000 \\
\hline
\end{tabular}

\section{References}

[1] Ahmad, I.A. (1975). A nonparametric test for the monotonicity of a failure rate function. Comm. Statist., 4, 967-974.

[2] Ahmad, I.A. (1994). A class of statistics of aging families of distributions with hypotheses testing applications. J. Statist. Plan. Inf., 4, 141-149.

[3] Ahmad, I.A. (1995). Nonparametric testing of classes of life distributions derived from a convex ordering. Parisankhyan Samikha, 2, 13-18.

[4] Ahmad, I.A. (1998). Testing whether a survival distribution is new better than used of an unknown specified age. Biometrka, 85, 451-456.

[5] Ahmad, I.A., Hendi, M., and Al-Nachawati (1999). Testing new better than used classes of life distribution derived from a convex ordering using kernel methods. J. Nonparam. Statistics, 11, 393-411.

[6] Barlow, R.E. and proschan, F. (1981). Statistical Theory of Reliability and Life Testing Probability Models. To begin with, Silver Spring, MD.

[7] Basu, S.K. and Ebrahimi, N. (1985). Testing whether survival functions is harmonic new better than used in expectation. Ann. Instit. Statist. Math., 37, 347-359.

[8] Borges W.D., Proschan, F. and Rodgrigues, J. (1984). A simple test for new better than used in expectation. Comm. Statist. A-Theory Methods, 13, 1216-1235.

[9] Cao, J.H. and Wang, Y.D. (1991). The NBUC and NWUC classes of life distributions. J. Appl. Probab., 28, 472-479.

[10] Deshpande, J.V. (1983). A class of tests for exponentiality against failure rate average. Biometrica, 70 (20), 514-518.

[11] Deshpande, J.V., Kochar, S.C. and Singh, H. (1986). Aspects of positive aging. J. Appl. Probab., 288, 773-779.

[12] Diab L.S., Kayid, M. and Mahmoud, M.A.W. (2009). Moments inequalities for NBUL distributions with hypotheses testing applications. Contem. Engin. Sci., 2 (7), 319-332.

[13] Diab, L.S. (2010). Testing for NBUL using goodness of fit approach with application. Stat. Papers, 51, 27-40.

[14] Ebrahimi, N. and Habbibullah, M., (1990). Testing whether the survival distribution is new better than used of specified age. Biometrika, 77, 212-215. 
M.A.W. Mahmoud et al.

[15] Elbatal, I., (2007). Some aging classes of life distributions at specific age. Int. Math. Forum, 2 (29), 1445-1456.

[16] Esary, J.D., Marshal., A. W. and Proschan, F., (1969). Shock models and wear process. Ann. Prob., 1, 627-649.

[17] Fisher, R.A. (1966). The Design of Experiments, Eight edition, Oliver \& Boyd, Edinburgh.

[18] Hendi, M.I, Al-Nachawati, H. and Montasser, M. and Al-Wasel, I.A. (1998). An exact for HNBUE class of life distributions. J. Satist. Comp. And simulation, 60, 261-275.

[19] Hollander, R.M., Park, D.H. and Proschan, F. (1986). A class of life distributions for aging. J. Amer. Statis. Assoc., 81, 91-95.

[20] Hollander, R.M. and Proschan, F. (1972). Testing whether new is better than used. Ann. Math. Statist., 43, 1136-1146.

[21] Hollander, R.M. and Proschan, F. (1975). Tests for mean residual life. Biometrika, 62, 585-592.

[22] Klefsjo, B., (1981). HNBUE survival under some shock models. Scand. J. Statis., 8, 39-47.

[23] Klefsjo, B., (1982). The HNBUE and HNWUE classes of life distribution. Naval Res. Logistic, 29, 331-344.

[24] Klefsjo, B. (1983). A useful aging property based on the Laplace transform. J. Appl. Probab., 20, 615626

[25] Koul, H.L. (1977). A new test for new is better than used. Commun. Statist. Theor. Meth., 6, 563-573.

[26] Koul, H.L. and Susarla, V. (1980). Testing for new better than used in expectation with incomplete data. J. Amer. Statist. Assoc., 75, 952-956.

[27] Kumazawa, Y. (1983). A class of tests statistics for testing whether new is better than used. Comm. Statist. Theor. Meth., 12, 311-321.

[28] Lee, A.J. (1990). U-statistics. Marcel-Dekker, New York.

[29] Mahmoud, M.A.W. EL-Arishy S.M. and Diab L.S. (2003). Moment inequalities for testing new renewal better than used and renewal new better than used classes. Int. J. Rel. Appl., 4, 97-123.

[30] Mahmoud, M.A.W. EL-Arishy S.M. and Diab L.S. (2005). Testing renewal new better than used life distributions based on u-test. Appl. Math. Model., 29, 784-796.

[31] Mahmoud, M.A.W. and Diab L.S. (2007). On testing exponentiality against HNRBUE based on a goodness of fit. Int. J. Rel. Appl., 8 (1), 27-93.

[32] Mahmoud, M.A.W., Abdul Alim, N.A. and Diab, L.S., (2009). On the new better than used renewal failure rate at specified time. Eqc., 24, 87-99.

[33] Parameshwar V. Pandit and M.P. Anuradha, (2007). On testing exponentiality against new better than used of specified age. Statistical Methodology, 4, 13-21.

[34] Rolski, T. (1975). Mean residual life. Bulletin of the International Statistical Institute, 4, 266-270. 